\title{
Epidemiologic and Clinical Features of Uveitis from a Tertiary Referral Hospital in Bulgaria from 2016
}

\author{
Gueorgui Markov* and Iva Petkova \\ Department of Ophthalmology, Medical University, Bulgaria
}

Submission: December 23, 2016; Published: January 10, 2017

*Corresponding author: Gueorgui Markov, Department of Ophthalmology, Alexandrovska Hospital, Medical University, Sofia, Bulgaria; Email: dr.georgimarkov@gmail.com

\section{Abstract}

Purpose: The aim of the present study is to describe the epidemiological and clinical features of recently presented patients with uveitis in a tertiary referral hospital in Bulgaria.

Material and methods: We conducted a retrospective observational study on the clinical records of 42 patients with uveitis of the Department of Ophthalmology, Alexandrovska Eye Hospital, Medical University - Sofia, Bulgaria. The period of the study was from March 2016 until December 2016. Follow-up ranged from 1 to 9 months. The diagnosis of the individual cases was based on a complete eye examination, review of systems, and additional laboratory and specialized testing methods.

Results: Forty-two new patients with uveitis presented to our clinic in 2016. Out of them, 28 (66.7\%) were female and 14 (33.3\%) - male (2:1 ratio). With the exception of one, who was racially mongoloid, all cases were white-caucasian. The age of the patients varied from 28 to 70 years, with a mean value of 51.9, median - 50.5, and mode of 40 years. Uveitis was bilateral in 25 (59.5\%) and monolateral in 17 (40.5\%) cases. We observed 17 cases $(40.5 \%)$ of anterior uveitis, 5 patients (11.9\%) with posterior, and $20(47.6 \%)$ with panuveitis. The major infectious etiologic agents were viruses from the herpes family, namely herpes simplex virus type -1 and varciella-zoster virus and toxoplasma gondii.

Conclusion: Uveitis comprises a diverse group of inflammatory conditions, which affect people of both genders and all ethnic groups at a relatively young and active age. Visual and anatomic outcomes may be excellent with timely diagnosis and treatment to prevent the development of vision-threatening complications. The epidemiologic data from this small cohort of patients from Bulgaria corresponds well with the already published literature for other different ethnic groups. The most common infectious etiologic agents for anterior and panuveitis belonged to the herpetic viruses family - $16.7 \%$. Non-infectious uveitis was associated most often with seronegative spondyloarthropathies and sarcoidosis. $47.6 \%$ of all cases were classified as idiopathic.

Keywords: Uveitis; Epidemiology; Panuveitis

\section{Introduction}

Uveitis is a broad concept, defining inflammation of the uveal tract, consisting of the iris, ciliary body, and choroid. Any compartment of the uvea may be affected distinctly or in association with the other parts and/or additional ocular structures [1,2]. Epidemiologically uveitis has been described in people of both genders, all ages, and in every racial group [13]. However, data regarding uveitis cases from Bulgaria, Europe, are lacking. The etiology is extremely diverse and there are many infectious and noninfectios conditions which may incite intraocular inflammation [1,2]. The presence of intraocular inflammation may lead to serious and irreversible visionthreatening structural and functional complications $[1,2,4]$.

\section{Purpose}

The aim of the present study is to describe the epidemiological and clinical features of recently presented patients with uveitis in a tertiary referral centre in Bulgaria.

\section{Material and Methods}

We conducted a retrospective observational study on the clinical records of 42 patients with uveitis of the Department of Ophthalmology, Alexandrovska Eye Hospital, Medical University - Sofia, Bulgaria. The period of the study was from March 2016 until December 2016. Follow-up ranged from 1 to 9 months. All uveitis patients who had presented prior to March 2016 were 
excluded from the study. The research was done with the consent and agreement of the Head of the Department of Ophthalmology. The diagnosis of the individual cases was based on a complete eye examination, review of systems, and additional laboratory and specialized testing methods. For data analysis, descriptive statistical methods were used.

\section{Results}

Forty-two new patients with uveitis presented to our clinic in 2016. Out of them, 28 (66.7\%) were female and 14 (33.3\%) - male (2:1 ratio). With the exception of one, who was racially mongoloid, all cases were white-caucasian. The age of the patients varied from 28 to 70 years, with a mean value of 51.9, median - 50.5, and mode of 40 years. Uveitis was bilateral in 25 (59.5\%) and monolateral in 17 (40.5\%) cases. From anatomical standpoint, we distinguished 17 cases $(40.5 \%)$ of anterior uveitis, 5 patients (11.9\%) with posterior, and 20 (47.6\%) with panuveitis. No cases of distinct intermediate uveitis were observed in this cohort.

With regard to anterior uveitis, there were 11 female $(64.7 \%)$ and 6 male patients (35.3\%). Bilateral involvement was noted in 8 cases (47.1\%) and monocular in 9 (52.9\%). The presenting age was from 30 to 78 , and most often 40 years (mean 55.9, median 58, mode 40). The inflammation was acute in $8(47.1 \%)$ patients (monocular involvement in all but one), recurrent - in 4 (23.5\%), and chronic in - 5 (29.4\%), most often bilateral. Etiologically, it was associated with herpetic infection in $3(17.6 \%)$ cases (keratouveitis), toxoplasmosis (manifesting as Fuchs heterochromic phenotype) - 1 (5.9\%), psoriasis - in $2(11.8 \%)$ (one with scleral involvement), Bechterew spondyloarthritis - in 1 (5.9\%), reactive arthritis - in 1 (5.9\%), cataract surgery - 1 (5.9\%), corneal trauma with vegetable matter - in 1 (5.9\%), antiglaucoma medications (dorzolamide/ timolol and brimonidine) -1 , and no definite extraocular cause was assumed in 6 (35.3\%). The visual acuity ranged between no light perception and $20 / 20$, most frequently - 20/20. The ocular complications observed were most commonly ocular hypertension in 4 patients $(23.5 \%)$, cataract - in $3(17.6 \%)$, macular oedema - in $2(11.8 \%)$, posterior synechiae- in 2 (11.8\%), corneal scarring - in 1 (5.9\%).

Concerning posterior uveitis, there were 3 female (60\%) and 2 male $(40 \%)$ patients. Bilateral involvement was noted in 3 cases $(60 \%)$ and monocular in $2(40 \%)$. The presenting age was from 28 to 50 (mean 39, median 40, mode - not applicable). The inflammation was chronic in 4 patients (80\%) and recurrent - in 1 (20\%). Etiologically all cases were idiopathic. Two male patients conformed to the punctate inner choroiditis (PIC) type of uveitis and one of them had Bechterew spondyloarthritis. One woman had focal granulomatous inflammation with adjacent vasculitis with evidence of old foci of inflammation in the retinal periphery. There was one case with bilateral serpiginous-like chorioretinitis with associated retinal optic nerve arteriolitis and one female with uveitis resembling either PIC or acute posterior multifocal pigment epitheliitis. Visual acuity varied from counting fingers to $20 / 20$, most commonly - 20/25. The major disturbing complaint was metamorhopsia. The ocular complications were most commonly intraretinal hemorrhages in 2 cases (40\%), atrophy of the retinal pigment epithelium and outer retinal layers- in 4 eyes (80\%), choroidal neovascular membrane and macular oedema - in 1 eye (20\%). From the associated ocular conditions, 4 patients (80\%) had myopia.

Panuveitis was encountered slightly more frequently than the other types, mostly because of our tertiary referral centre status. There were 14 female $(70 \%)$ and 6 male $(30 \%)$ patients. Bilateral involvement was noted in $14(70 \%)$ and monocular in 6 (30\%) of the cases. The presenting age was from 30 to 76 - mean 51.7 years. The inflammation was acute in $1(5 \%)$, recurrent - 8 (40\%), and chronic in 11 cases (55\%). Final visual acuity ranged between no light perception and 20/20, on average 20/50. Etiologically we found associations with herpetic infection in 4 patients $(20 \%)$, sarcoidosis - in $4(20 \%)$, toxoplasmosis in 1 (5\%), Behcet disease - in 1 (5\%), malignant hypertension (masquerade syndrome) - in 1 (5\%), and the remaining 9 $(45 \%)$ were designated as idiopathic. The ocular complications included - cataract - in 7 patients (35\%), preretinal membrane - in 6 (30\%), ocular hypertension - in 5 (25\%), macular oedema - in 4 (20\%), secondary glaucoma - in 3 (15\%), retinal detachment - in 3 (15\%), vireopapillary traction syndrome - in 3 (15\%), posterior synechiae - in $3(15 \%)$, extensive chorioretinal atrophy - in 2 (10\%), anterior synechiae - in 1 (5\%), optic nerve atrophy - in $1(5 \%)$, vitreoretinal traction syndrome - in $1(5 \%)$, hypotony - in $1(5 \%)$.

\section{Discussion}

In general, uveitis was not a common diagnosis at our institution for a period of 9 months. In terms of gender and age, our data correspond to the already published figures, indication overall female preponderance $[1,5,6]$ and onset most frequently around the age of $40[1,2,7,8]$. This was especially true for the cases of anterior and panuveitis, regardless of the diverse etiology. Anterior uveitis is usually the most common type of intraocular inflammation reported worldwide [1,2]. In the present study, however, panuveitis was the most frequent uveitis entity at $47.6 \%$, but we assume this to be due to referral bias.

Concerning the causative factors the major infectious agents were viruses from the herpes family, namely Herpes simplex virus type -1 and Varciella-zoster virus, and toxoplasma gondii. In those cases the diagnosis was corroborated by typical clinical findings, polymerase chain reaction of aqueous humour, and the response to therapy. With regard to noninfectious causes of uveitis, seronegative spondyloarthropathies were associated with 4 cases of anterior uveitis, sarcoidosis - with 4 patients with panuveitis, and Behcet disease with 1 patient with bilateral generalized intraocular inflammation. Secondary to failure to identify definitive etiologic factors, many cases were classified as being idiopathic - 6 with anterior uveitis (35\%), all 5 with 
posterior (100\%), and 9 with panuveitis (45\%). All in all, 20 cases were idiopathic (47.6\%). There was a relatively high frequence of myopia in the patients with posterior uveitis in our group, which had also been described for similar cases by other authors [1].

The visual prognosis was excellent in the cohort with anterior uveitis and it was twice as worse and guarded in those with panuveitis. Timely and sensible treatment were the necessary conditions for a good outcome. The reason for the poorer vision in the panuveitis group was largelly due to a significant delay in the institution of systemic therapy, including immunomodulatory medications, which had led to the development of irreversible vision-limiting complications.

The ocular complications of uveitis were not infrequent and often there were multiple alterations in a single patient. Structural and functional complications were observed more frequently in the panuveitis group and in the whole cohort consisted predominantly of cataract $(23.8 \%)$, ocular hypertension $(21.4 \%)$, macular oedema $(16.7 \%)$, and preretinal membrane (14.3\%). Some of those would necessitate additional conservative and surgical treatment. Albeit the small size of the analyzed sample, the relative percentages of the various complications are very similar to the reported in the literature for other populations $[1,4,7-11]$.

\section{Conclusion}

Uveitis comprises a diverse group of inflammatory conditions, which affect people of both genders and all ethnic groups at a relatively young and active age. Visual and anatomic outcomes may be excellent with timely diagnosis and treatment to prevent the development of vision-threatening complications. The epidemiologic data from this small cohort of patients from
Bulgaria corresponds well with the already published literature for other different ethnic groups. The most common infectious etiologic agents for anterior and panuveitis belonged to the herpetic viruses family - $16.7 \%$. Non-infectious uveitis was associated most often with seronegative spondyloarthropathies and sarcoidosis. $47.6 \%$ of all cases were classified as idiopathic.

\section{References}

1. Foster CS, Vitale A (2012) Diagnosis and Treatment of Uveitis Second Edition. Jaypee Brothers Medical Publishers, New Delhi, India.

2. Intraocular Inflammation and Uveitis. 2013-2014 Basic and Clinical Science Course. AAO, San Francisco, USA.

3. Biswas J, Narain S, Das D, Ganesh SK (1996) Pattern of uveitis in referral uveitis clinic in India. Int Ophthalmol 20(4): 223-228.

4. De Smet M, Okada A (2010) Cystoid macular edema in uveitis. Dev Ophthalmol Basel, Karger, 47: 136-147.

5. Islam SM, Tabbara KF (2002) Causes of uveitis at The Eye Center in Saudi Arabia: A retrospective review. Ophthal Epidemiol 9(4): 239249.

6. Suhler EB, Lloyd MJ, Choi D, Rosenbaum JT, Austin DF (2008) Incidence and prevalence of uveitis in Veterans Affairs Medical Centers of the Pacific Northwest. Am J Ophthalmol 146(6): 890-896.

7. Nussenblatt RB, Whitcup SC, Uveitis (2010) Fundamentals and Clinical Practice Philadelphia, Elsevier, Netherlands.

8. Prete M, Guerriero S, Dammacco R, Fatone MC, Vacca A, et al. (2014) Autoimmune uveitis: a retrospective analysis of 104 patients from a tertiary reference center. J Ophthalmic Inflamm Infect 24(4): 17.

9. Rothova A (2007) Inflammatory cystoid macular edema. Curr Opin Ophthalmol 18(6): 487-492.

10. Sallam A, Sheth HG, Habot-Wilner Z (2009) Outcome of raised intraocular pressure in uveitic eyes with and without a corticosteroidinduced hypertensive response. Am J Ophthalmol 148(2): 207-213.

11. Sonam AB, Kumar V, Raina U, Ghosh B, Thakar M (2011) Inflammatory glaucoma. Oman J Ophthalmol 4(1): 3-9.

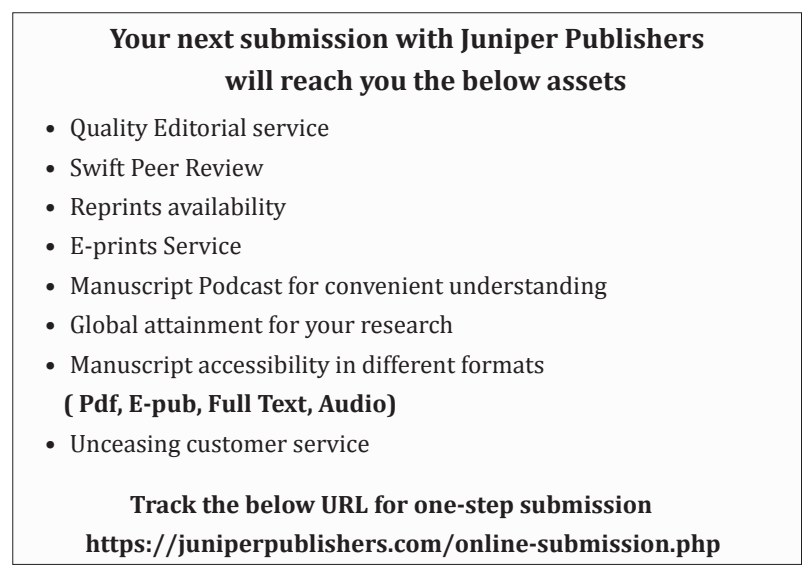

